\title{
Electrical Impedance and Modulus Characteristics of Bulk PZT Ceramics Modified by Mn and Ce
}

\author{
Balgovind Tiwari ${ }^{1}$, T. Babu ${ }^{2}$, R.N.P. Choudhary ${ }^{3}$ \\ ${ }^{1}$ Assistant Professor, Dept. of Physics, IIIT-RKValley, RGUKT, A.P., India \\ ${ }^{2}$ Teacher, Dept. of Physics, Sri Chaitanya High School, Vempalli, Kadapa, A.P., India \\ ${ }^{3}$ Professor, Dept. of Physics, ITER-SOA University, Orissa, India.
}

\begin{abstract}
The compounds of $\mathrm{Pb}\left(\mathrm{Zr}_{0.35-x} Y_{x} \mathrm{Ti}_{0.65}\right) \mathrm{O}_{3}(x=0.00,0.10$ and $Y=\mathrm{Mn} / \mathrm{Ce})$ stoichiometry were prepared by $a$ standard solid state route of mixing. The lead zirconatetitanate (PZT) has been modified by substituting $10 \%$ of manganese $\left(\mathrm{Mn}^{+4}\right)$, and cerium $\left(\mathrm{Ce}^{+4}\right)$ ions, by replacing zirconium $\left(\mathrm{Zr}^{+4}\right)$. The effect of substitution of $\mathrm{Mn}^{+4}$ and $\mathrm{Ce}^{+4}$ ions, on the electrical properties of PZT based ceramics, has been investigated systematically in this work. To study the impedance and modulus characteristics, adopted are the complex impedance and complex modulus spectroscopic methods respectively. Results indicated that the compounds exhibit negative coefficient of resistance behavior (as evident from impedance spectra), and real ionic conductor nature (as evident from modulus spectra). The electrical properties of the compounds are primarily dominated by the grains rather than grain boundaries and electrode-material interface polarization.
\end{abstract}

Keywords: Ferroelectric, Impedance, Modulus, Relaxation

\section{Introduction}

PZT, a well-known piezoelectric, is one of the potential ceramic for the use in various electronic applications such as sensors, actuators, memories [1], etc [2-3]. The vast usage of PZT in the above mentioned applications is due to its prodigious electrical properties such as high dielectric permittivity, large electrical resistivity, etc [4]. The unit cell of PZT consists of large sized cations $\left(\mathrm{Pb}^{+2}\right)$ and small sized cations $\left(\mathrm{Zr}^{+4} / \mathrm{Ti}^{+4}\right)$ at $\mathrm{A}$ and $\mathrm{B}$ sites respectively [5]. While the oxygen $\left(\mathrm{O}^{-2}\right)$ atoms are situated at the face centers of unit cells, in the form octahedron arrangement [6]. Since the electrical properties can be altered through various ways such as doping/modification [3, 6], and varying synthesis techniques [6-8], etc, PZT is being investigated and used broadly. Doping and/or modification can be done through the introduction of foreign element ions in the lattice of PZT. One can choose the elements of valence ranging from +1 to +3 and +3 to +6 , for the suitable modification at $A$ and $B$ sites respectively [9].

Due to the crucial role of PZT in electronic applications, it is essential to investigate the electric properties of PZT. Because PZT is primarily considered as a grain-grain boundaryelectrode material interface system $[3,10]$. Studying the interplay between grains and grain boundaries, and their contribution on the electrical properties helps to produce the ceramics with desired properties [3]. Also the grain boundaries could be often active, when electrical conduction is taking place in electroceramic, and may also act as barriers that hinders the movement of charge carriers $[4,11]$. One of the best technique, to study 
the aforementioned contribution, is complex impedance spectroscopy (CIS) [2-3]. The information on the occurrence of electrical process in a polycrystalline ceramic oxide, can be extracted through CIS by applying ac electrical filed externally. The advantages (of using CIS) include the availability of the instruments and the ease of their use, as well as the fact that the experimentalist can control the frequency range. The measurement of impedance helps to find its real and imaginary parts ( $Z^{\prime}$ and $\left.Z^{\prime \prime}\right)$ from the following expressions $[3,12]: Z^{*}=Z^{\prime}-j Z^{\prime \prime}$, where $Z^{\prime}=\frac{R}{1+(\omega C R)^{2}}, Z^{\prime \prime}=\frac{\omega C R^{2}}{1+(\omega C R)^{2}}$, where $\omega, \mathrm{C}$ and $\mathrm{R}$ are the angular frequency, capacitance and resistance of the material. The values of $Z^{\prime}$ and $Z^{\prime \prime}$ can used further, to evaluate real and imaginary parts $\left(\mathrm{M}^{\prime}\right.$ and $\left.\mathrm{M}^{\prime \prime}\right)$ of electric modulus as per the following expressions [2-3]: $\mathrm{M}^{*}=\mathrm{M}^{\prime}+\mathrm{j} \mathrm{M}^{\prime \prime}=1 / \varepsilon^{*}=$ $j \omega \varepsilon_{0} Z^{*}, M^{\prime}=\omega C_{0} Z^{\prime \prime}, M^{\prime \prime}=\omega C_{0} Z^{\prime}$, where $\varepsilon^{*}$ is complex permittivity and $j=\sqrt{-1}$.

The novelty of our study is to synthesize the $\mathrm{Mn}$ and $\mathrm{Ce}$ modified (i.e. PZMT and PZCT) PZT ceramics and investigate the impedance (resistive) and modulus (capacitive) characteristics. To do so, solid state reaction has been adopted for the preparation of the samples, as it is fast and cost effective method. Then electrical measurements were performed under ambient atmosphere, to investigate the electrical characteristics such as complex impedance and modulus planes, spectroscopic plots (such as $Z^{\prime}$ and $Z^{\prime \prime}$ vs. frequency, and $\mathrm{M}^{\prime}$ and $\mathrm{M}^{\prime \prime}$ vs. frequency).

\section{Experimental Section}

The chemicals of $99.9 \%$ purity have been used for the preparation of samples. Inorganic oxides such as lead oxide (M/S. LobaChemiePvt. Ltd., India), zirconium dioxide (M/S. Sarabhai Chemicals, India), titanium dioxide (M/S. LobaChemiePvt. Ltd., India), manganese dioxide (M/S. Sarabhai Chemicals, India) and cerium dioxide (M/S. LobaChemiePvt. Ltd., India) were taken in desired stoichiometry (i.e. $\mathrm{Pb}\left(\mathrm{Zr}_{0.35}\right.$ $\left.{ }_{x} \mathrm{Y}_{\mathrm{x}} \mathrm{Ti}_{0.65}\right) \mathrm{O}_{3}(\mathrm{x}=0.00,0.10$ and $\left.\mathrm{Y}=\mathrm{Mn} / \mathrm{Ce})\right)$. The detailed step by step procedure and the complete process parameters, regarding the preparation of samples, have been reported elsewhere $[3,5]$.

\section{Results and Discussion}

\subsection{Complex Impedance Spectrum}

Figure 1 and its insets represent the complex impedance plots of the PZT, PZMT and PZCT ceramics. The appearance of high frequency/first and start of second semicircular arcs, for PZT and PZMT, suggests the probable contribution of grains and grain boundaries towards the electrical properties. But the presence of single semicircular arc indicate that the electrical properties of PZCT compound is mainly governed by grains [2]. The absence of third semicircular arc, for all the compounds, indicate the negligible contribution of electrode-material interface polarization. All the arcs in the spectrum are depressed, with centers lying below the $Z^{\prime}$ axis, indicating the occurrence of non-Debye kind of electrical relaxation phenomenon. Hence our impedance data may satisfy Cole-Cole relaxation model due to the appearance of distorted/depressed semicircular arcs [3]. The magnitude of bulk resistance $\left(R_{b}\right)$ is slightly decreased and largely enhanced with the substitution of $\mathrm{Mn}$ and $\mathrm{Ce}$ ions respectively. Further, the magnitude of $\mathrm{R}_{\mathrm{b}}$ of each sample is decreasing with increase in temperature indicating semiconducting behavior of the compounds at higher temperatures.
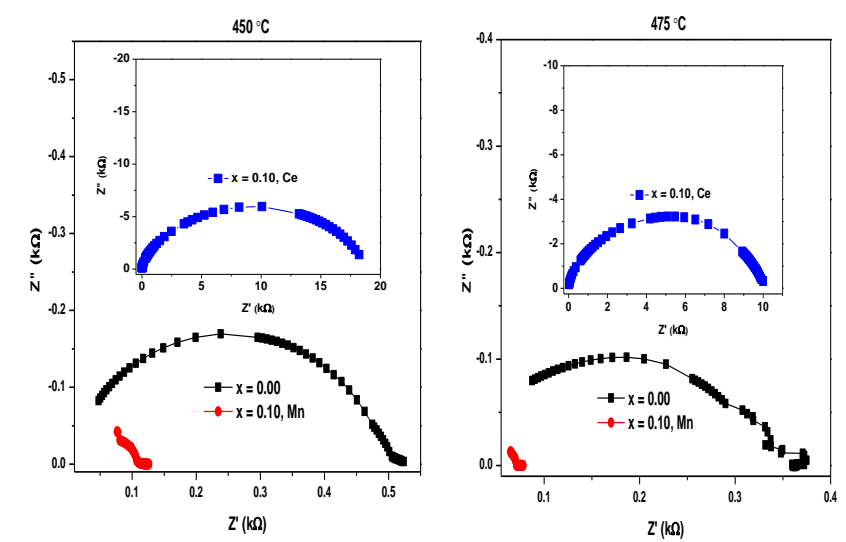

Fig.1. Nyquist Plots

\subsection{Variation of real part of impedance with frequency}

Figure 2 and its insets shows the nature of variation of $Z^{\prime}$ of PZT, PZMT and PZCT ceramics with frequency, at higher temperatures. The higher values of $Z^{\prime}$, for all samples, in the region of low frequency is due to the presence of all types of 
polarizations in the compounds. The lower values of $Z^{\prime}$, for all samples, in the region of high frequency is due to the reduced barrier properties (as a result of release of space charge polarization) of the compounds. The decrease in the magnitude of $\mathrm{Z}^{\prime}$ with increase in frequency, indicate the possible rise in ac conductivity of the samples [3]. The magnitude of $Z^{\prime}$ is slightly and largely enhanced with the substitution of $\mathrm{Mn}$ and $\mathrm{Ce}$ ions respectively. Further, the magnitude of $Z^{\prime}$ of each sample is decreasing with increase in temperature, indicating semiconducting behavior of the compounds at higher temperatures [2].
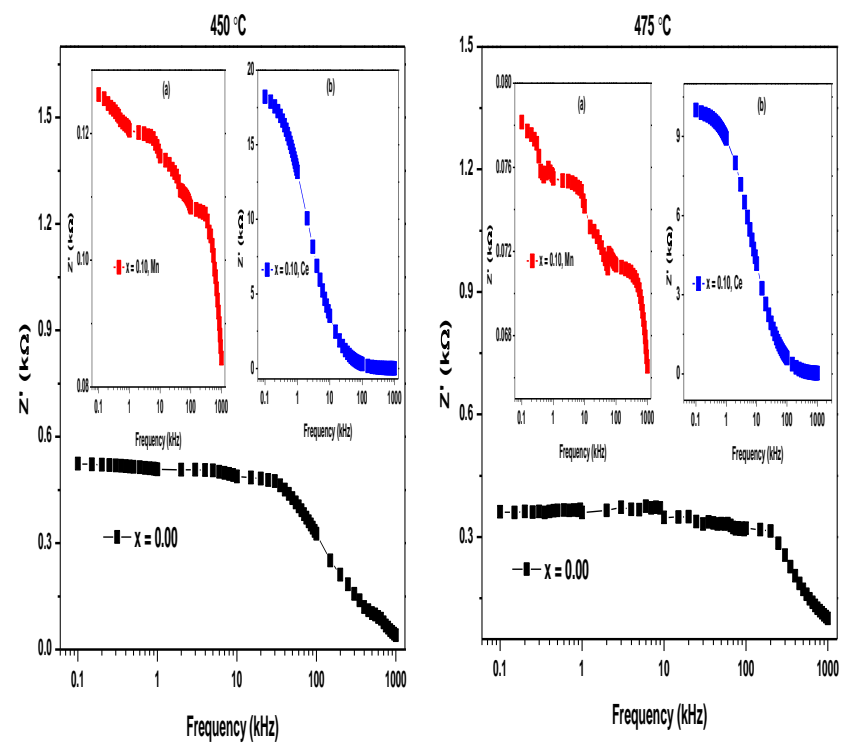

Fig.2. Variation real part of impedance with frequency

\subsection{Variation of imaginary part of impedance with frequency}

Figure 3 and its insets show the nature of variation of Z" (impedance loss) of PZT, PZMT and PZCT ceramics with frequency. The presence of peaks in the spectrum indicate the occurrence of electrical relaxation phenomenon in the compounds [3], except PZMT, for which the peak may form beyond our measurement strength. The increase in the peak width of PZT with the substitution of Ce ions, indicate the distribution of relaxation time with different time value constants. The change in position of the peaks, for each sample, indicate that the relaxation is strongly dependent on temperature. The reduction in the values of $Z^{\prime \prime}$, at high frequency region, indicate the presence of space charge polarization in the compounds [3].
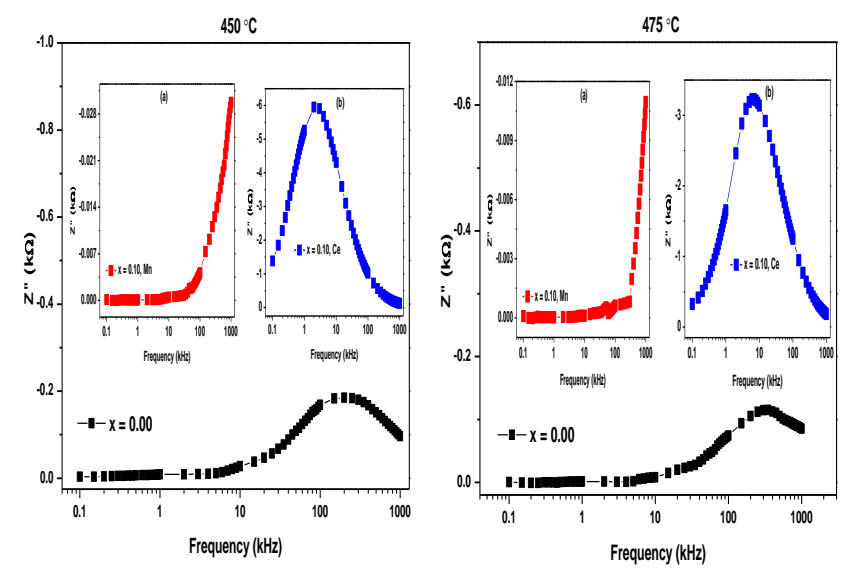

Fig.3. Variation of imaginary part of impedance with frequency

\subsection{Complex Modulus Spectrum}

Figure 4 represent the complex modulus plots (i.e. variation of real part of modulus with imaginary part of modulus) of PZT, PZMT and PZCT ceramics. The appearance of single semicircular arcs in the spectrum, confirms the domination of grains on the electrical properties of compounds [12]. The non-presence of second semicircular arc, confirmed that the electrical properties of the compounds have negligible contribution of electrode-material interface polarization. The arcs are asymmetric indicating the distribution of relaxation time with different time constants. Hence, non-Debye electrical relaxation is confirmed.
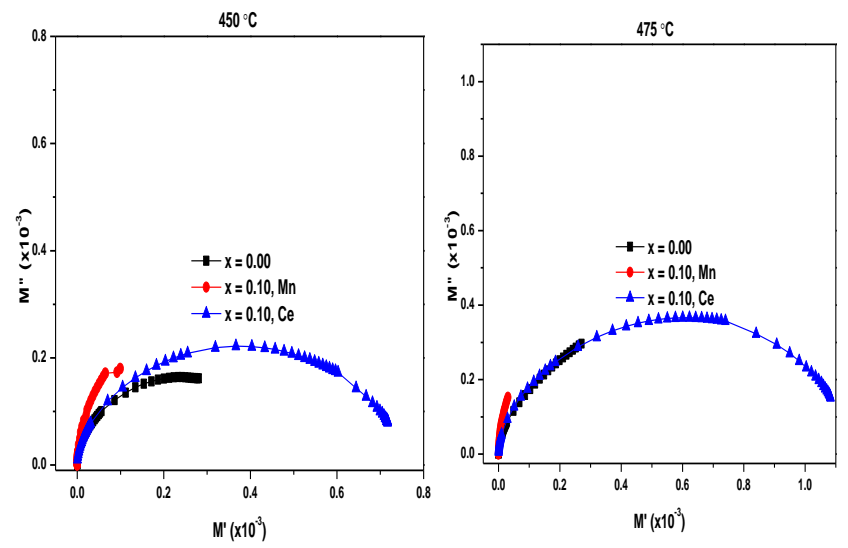

Fig.4. Complex modulus spectrum

\subsection{Variation of real part of modulus with frequency}

Figure 5 shows the nature of variation of $\mathrm{M}^{\prime}$ of PZT, PZMT and PZCT ceramics with frequency. In the region of low frequency, the magnitude of 


\section{www.rspsciencehub.com}

$\mathrm{M}^{\prime}$ is approximately reaching the value of zero. With rise in frequency, the value of $\mathrm{M}^{\prime}$ of all compounds is increasing, indicating the existence of electrical conduction due to the motion of charge species over small distances [3].
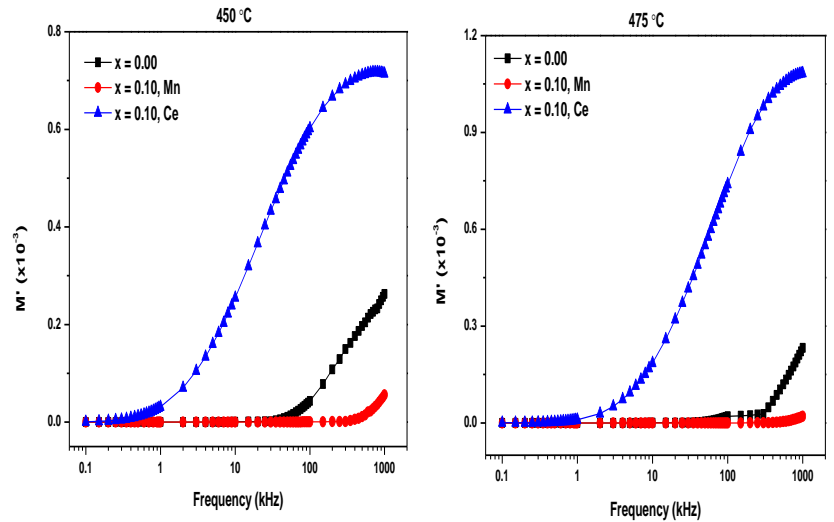

Fig.5. Variation of real part of modulus with frequency

\subsection{Variation of imaginary part of modulus with frequency}

The relaxation of dielectric can be characterized with the help of varying nature of $\mathrm{M}^{\prime \prime}$ with frequency, as shown in Figure 6. The appearance of peak, for PZCT, indicate the dielectric relaxation phenomenon. For PZT and PZMT compounds, the peaks might have formed beyond our measurement domain. The position of peak of PZCT, is shifted towards the region of high frequency with rise in temperature.
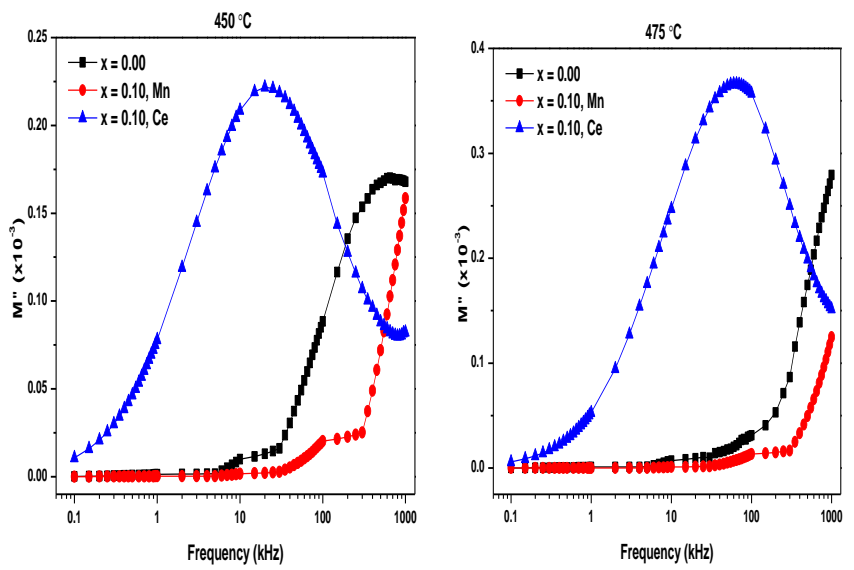

\section{Fig.6. Variation of imaginary part of modulus with frequency}

This confirms the dependence of dielectric relaxation on temperature. Also the presence of peak in the spectrum, confirm the negligible
Volume 02 Issue 09 September 2020

contribution of electrode-material interface polarization towards the electrical properties of the compounds. Further, the peaks are not symmetric indicating the distribution of relaxation time with different time value constants $[2,3]$. Hence, nonDebye kind of electrical relaxation is confirmed.

\section{Conclusions}

A systematic investigation on the electrical properties of Mn and Ce modified PZT ceramics, has been carried out in this work. Complex impedance spectrum indicated the effect of grains on the electrical properties, which has been further confirmed through complex modulus spectrum. The effect arising from electrode-material interface polarization is negligible, as indicated from both impedance and modulus spectra. The impedance data of the samples may satisfy the Cole-Cole kind of electrical relaxation, due to the asymmetric, depressed and distorted nature of the curves (appeared in complex impedance plots). The nature of modulus spectra indicated that the electrical conduction in the compounds, is mainly due to the hopping of charge species over small distances. Moreover, the increase in height of $\mathrm{M}^{\prime \prime}$ peaks with increase in temperature suggested the real ionic conductor behaviour of the compounds.

\section{Acknowledgement}

We (authors) are grateful for the experimental facilities provided by IIT Kharagpur. We also thak the partial financial support of DST-SERB.

\section{References}

[1] Zhang Qi, Whatmore R.W. (2004). Low fatigue lead zirconatetitanate-based capacitors modified by manganese for nonvolatile memories, Materials Science and Engineering B, 109, 136-140.

[2] Balgovind Tiwari andChoudharyR.N.P. (2010).Frequency-temperature response of $\mathrm{Ce}$ modified $\mathrm{Pb}\left(\mathrm{Zr}_{0.65-\mathrm{x}} \mathrm{Ce}_{\mathrm{x}} \mathrm{Ti}_{0.35}\right) \mathrm{O}_{3}$ ferroelectric ceramics: Impedance spectroscopic studies, Journal of Alloys and Compounds, 4931 (2), 1-10. 
[3] Balgovind Tiwari, Babu $\mathrm{T}$ and ChoudharyR.N.P. (2020). AC Impedance and Modulus Spectroscopic Studies of $\mathrm{Pb}\left(\mathrm{Zr}_{0.35-\mathrm{x}} \mathrm{Ce}_{\mathrm{x}} \mathrm{Ti}_{0.65}\right) \mathrm{O}_{3} \quad(\mathrm{x}=0.00, \quad 0.05$, $0.10, \quad 0.15) \quad$ Ferroelectric Ceramics, Materials Chemistry and Physics, 256, 123655.

[4] Balgovind Tiwari (2011). Ph.D. Thesis, IIT Kharagpur, India.

[5] Balgovind Tiwari, Babu $\mathrm{T}$ and ChoudharyR.N.P. (2020).Synthesis of $\mathrm{Pb}\left(\mathrm{Zr}_{0.35-\mathrm{x}} \mathrm{Mn}_{\mathrm{x}} \mathrm{Ti}_{0.65}\right) \mathrm{O}_{3}, \mathrm{x}=0.00,0.02,0.06$, 0.10 ceramics and their structural, dielectric characteristics, 7, 055701.

[6] Chen B, Wu L, Chure $M$ and Chen Y (2010). Fabrication of PZT by sol-gel method, Proceedings of the 2010 Symposium on Piezoelectricity, Acoustic Waves and Device Applications, Xiamen, 310-314.

[7] Balgovind Tiwari, Babu $T$ andChoudharyR.N.P. (2020). Effect of Manganese Doping on Dielectric Characteristics of Lead ZirconateTitanate of Different Zirconium/Titanium Ratios, IOP Conference Series: Materials Science and Engineering, 764, 012029.

[8] Prakash C and JunejaJ.K. (2006). Investigations on $\mathrm{Sm}$ and $\mathrm{Nb}$ substituted PZT ceramics, Modern Physics Letters B, 20, 1879-1882.

[9] NiranjanSahu (2011).Ph.D. Thesis, NIT Rourkela, India.

[10]MoulsonA.J. and Herbert J.M., Electroceramics, $2^{\text {nd }}$ Edition, England: John Wiley \& Sons Ltd., (2003).

[11]Buchanan R.C., Ceramic Materials for Electronics, $3^{\text {rd }}$ Edition, New York: CRC Press, (2004).

[12]Callister W.D., Materials Science and Engineering: An Introduction, $7^{\text {th }}$ Edition, New York: John Wiley \& Sons Ltd., (2007). 